\title{
CRISPR-Cas System: A Brief History and Future in the Diagnosis and Treatment of Infectious Diseases
}

Barış Otlu

Department of Medical Microbiology, Inönü University School of Medicine, Malatya, Turkey

T he discovery of Clustered Regularly Interspaced Short Palindromic Repeat (CRISPR) and CRISPR-Associated System (Cas) have led to revolutionary advancements in almost every field of biology. The CRISPR-Cas system is part of an adaptive immune system in bacteria, and this system protects the organism from invading genetic elements of bacteriophages and plasmids. In this article, the discovery of the CRISPR-Cas system, its mechanism of action, and particularly its use in infectious diseases will be discussed.

\section{BRIEF HISTORY}

Though the CRISPR sequence was first recognised in the alkaline phosphatase (iap) gene of Escherichia coli (E. coli) in 1987, the biological function of these unusual repeat sequences has long been undetermined (1). Francisco Mojica was the first scientist who characterised the CRISPR sequences. Francisco Mojica and his colleague Ruud Jansen used the term "CRISPR" in their personal communications, then Jensen first used this term in one of his articles in 2002 (2). Finally, Mojica and et al. (3) showed that these repeat sequences matched with the genome parts of bacteriophages, plasmids, and prophages, and the authors developed a thesis projecting that CRISPR was the adaptive immune system of bacteria. In the same year, two distinct research groups published similar results from their work performed on the genomes of Yersinia pestis and Streptococcus thermophilus (S. thermophilus) $(4,5)$. Furthermore, in the S. thermophilus study, a shared gene sequence was also defined on the forward sequences of all spacers, homologues to that of the viral genes. This sequence-essential for recognising the target-was named as the protospacer adjacent motif (PAM) sequence (5).

In parallel studies, genes encoding DNA-repair proteins specific for hyperthermophilic archea, were shown to be related to CRISPR, and were later defined as the cas (CRISPR-associated) genes (6). Cas proteins have helicase and nuclease functions, hence their ability to unwind and cut the targeted DNA segments. Comparative analyses of the genome indicated that CRISPR and cas proteins work together, and prokaryotic cells
Corresponding Author: Barış Otlu

\section{E-mail:}

botlu@yahoo.com

Received: March 29, 2019 Accepted: April 19, 2019 Published: May 30, 2019

\section{Suggested Citation:}

Otlu B. CRISPR-Cas System: A Brief History and Future in the Diagnosis and Treatment of Infectious Diseases. Infect Dis Clin Microbiol 2019; 1: 2-5.

DOI: $10.5152 / \mathrm{idcm} .2019 .19007$ 
have an acquired immune system similar to that of the RNA-interference (RNAi) seen in eukaryotic cells (7).

Significant advancements have been gained from studies in industrial microbiology for a better understanding of the CRISPR system. Bacteria are extensively used in fermentation processes and biotechnology. However, the bacteria used in industrial applications are extremely sensitive to phage attacks, and this situation may affect production. In a recent investigation, the response of $S$. thermophilus, a common organism used in yoghurt and cheese production, to a bacteriophage attack was studied. In this experimental study; following the phage attack, new spacers produced from the phage genomic sequences were integrated into the bacterial genome, and the phage resistance phenotype of the cell could be changed by the addition or removal of these sequences (8). By this way, the new phage sequences inserted into the CRISPR locus (added to the genomic library) were shown to have a protective role in subsequent phage attacks, namely the adaptive immunity of the bacteria.

One of the major questions regarding the CRISPR-cas system was related to the mechanism by which cas proteins were directed to their targets. Firstly, the spacer sequences in the CRISPR system, which arise from the phage genome, were transcribed with small RNAs, known as crRNA that were responsible for guiding the cas proteins to the target DNA (9). From the studies on the CRISPR-Cas9 system, in addition to the crRNA, a second small trans-activating CRISP RNA (tracrRNA) was discovered in 2011. Both RNAs formed a duplex which directed the Cas9 to its target (10).

Jinek et al. (11) showed that the crRNA and tracrRNA are complementary to each other, a finding that may be the most substantial advancement after the discovery of the CRISPR-Cas system. This double-stranded guide directs Cas9 from PAM to the invading DNA and mediates its cutting. The investigators designed a new molecule called guide RNA (gRNA) by combining the crRNA and tracrRNA. This discovery facilitated the targeted recognition and guided cleavage at any position on the genome.

\section{DIAGNOSTIC AND THERAPEUTIC USE IN INFECTIOUS DISEASES}

After the first realisation of its potency in genomic engineering, the CRISPR-Cas system was after that considered to be the excellent tool for genomic manipulations due to its easy-to-design and easy-touse properties (12). It exhibited an almost four-fold improved efficiency compared with previous approaches utilised for genomic manipulations, such as ZFN (zinc-finger nucleases) and TALEN (transcription activator-like effector nucleases). Because of these advantages, the CRISPR-Cas system has become widely used for diagnostic and therapeutic purposes (13).

The CRISPR-Cas system has expanded our understanding of microbe-host interactions and provided some important benefits for the treatment and diagnosis of infections. Today, multidrug-resistant pathogens and persistent viral infections such as HSV, HIV, and Hepatitis B virus (HBV) are the main interest areas for CRISPR-based infectious disease therapeutics.

Recently, silencing the antimicrobial resistance and restoration of antimicrobial susceptibilityand virulence genes of bacteria could be achieved using the CRISPR technology, without harming the normal microbiota (14). Citorik et al. (15) recently demonstrated successful elimination of the eae gene- a gene necessary for the intimate attachment to epithelial cells-of enterohemorrhagic $E$. coli (EHEC) with the RNA-guided Cas9 system. In that study, the RNA-guided Cas9 system was delivered to EHEC by a phagemid. With this approach used in the EHEC-infected Galleria mellonella larvae model, the number of bacteria was reduced by approximately 20-fold. Additionally, it was shown that the genes encoding beta-lactamase and carbapenemase enzymes could be inhibited in a mixed organism population in an agent-specific manner $(14,15)$. Considering the progressively increasing antimicrobial resistance, using CRISPR technology to manipulate the susceptibility patterns of the infecting pathogens to the antimicrobials before or during treatment may be possible in the near future. 
A number of viral pathogens produce persistent infections by integrating their genome episomally or into the human chromosomal DNA during infection. Both in vitro and animal model studies have provided substantial data that highlight the CRISPR technology as a promising therapeutic method which can be used to eliminate or reduce chronic and resistant viral infections. There are a number of experimental studies in the literature, which have shown that proviral DNA, integrated into the host's genome following acute HIV infection, can be cleared by the CRISPR technology or have its expression reduced. Yin et al. (16) achieved the elimination of HIV proviral DNA from neural progenitor cells in 2017. In that study, the researchers used an adeno-associated virus (AAV) carrying multiple sgRNAs and S. aureus Cas9. In a similar study, HIV-1 proviral DNA was cleared from infected blood mononuclear cells by using a lentivirus vector in a humanised mice model (17).

Similar studies were also performed for Herpes viruses such as HSV-1, CMV, and EBV. In these studies, viral genes could be eliminated from the host genome using the CRISPR-Cas9 system and gRNA targeting. Van Diemen et al. (18) successfully removed $95 \%$ of the target viral genetic elements of EVB, which were essential for virus fitness, and its nuclear antigen-1 (EBNA-1) from latently-infected Burkitt lymphoma cells (18).

The CRISPR-Cas system has also been studied for the treatment of HBV which has infected about 250 million people all over the world and causes approximately 1 million deaths annually. Existing therapeutic strategies for HBV infection can not clear the covalently closed circular DNA (cccDNA) in HBV which is found episomally in the hepatocytes and provides the persistent infection of the virus. Effective and accurate treatment of HBV infection requires novel therapeutic approaches targeting the blockade or complete removal of persisting cccDNA in these cells (19). In another study, Zheng $Q$ et al. (20) achieved an increase in the inhibition of the total duck hepatitis B virus (DHBV) DNA in infected duck hepatocytes with the CRISPR-Cas9 system (20).

In recent years, CRISPR-Cas based systems for the di- agnosis of infectious diseases, surveillance of emerging pathogens, microbial genotyping, and antimicrobial resistance detection have been developed (21). For the diagnosis of infectious diseases, newer applications of the CRISPR system are being used, such as "collateral cleavage" mediated by Cas12 and Cas13 nucleases. These applications are generally based on the cutting principle of a designed RNA using the CRISPR-Cas system, which is a probe-like structure having reporter and quencher molecules on both sides. When the designed CRISPR-Cas system binds to the target sequence, the reporter RNAs are cut by the "collateral cleavage" effect of the Cas13 endonuclease, separating the reporter and quencher from each other, and hence allowing the signal from the reporter molecule to be measured $(14,22,23)$.

The first platform produced was named "Specific High Sensitivity Enzymatic Reporter Unlocking" (SHERLOCK). This platform uses Cas13a cleavage which is developed following the isothermal recombinase amplification (RPA) or reverse transcription (RT) RPA. Gootenberg and et al. (22), using the SHERLOCK platform, could directly detect Zika and Dengue viruses from saliva in less than two hours. The researchers could also detect some bacterial pathogens including E. coli and P. aeruginosa, and further showed some resistance genes (KPC and NDM) in K. pneumoniae. Additionally, the authors emphasized that this system was very sensitive and was able to detect single nucleotide polymorphisms (SNP). A second platform that was developed using a similar method, the "DNA Endonuclease Targeted CRISPR Trans Reporter" (DETECTR), utilizes Cas12a enzymatic activity in isothermal RPA. In this platform, crRNA-Cas12a complex binds to target DNA and cuts the fluorescent reporter of the bonded ssDNA unselectively. With this platform, HPV-16 and HPV-18 could be detected in clinical specimens within one hour (24).

Dozens of advancements have been gained during the discovery process of a bacterial immune system which has evolved to a revolutionary genome manipulation tool. It is very clear that the CRISPR-Cas technology will bring substantial developments not only in synthetic biology, but also in genetic engineering, biotechnology, personalized medicine, cell biology, and agriculture and farming. 


\section{REFERENCES}

1 Ishino Y, Shinagawa H, Makino K, Amemura M, Nakata A. Nucleotide sequence of the iap gene, responsible for alkaline phosphatase isozyme conversion in Escherichia coli, and identification of the gene product. J Bacteriol 1987; 169: 5429-33.

2 Jansen R, van Embden JD, Gaastra W, Schouls LM. Identification of a novel family of sequence repeats among prokaryotes. OMICS 2002; 6: 23-33.

3 Mojica FJ, Díez-Villaseñor C, García-Martínez J, Soria E. Intervening sequences of regularly spaced prokaryotic repeats derive from foreign genetic elements. J Mol Evol 2005; 60: 174-82.

4 Pourcel C, Salvignol G, Vergnaud G. CRISPR elements in Yersinia pestis acquire new repeats by preferential uptake of bacteriophage DNA, and provide additional tools for evolutionary studies. Microbiology 2005; 151: 653-63.

5 Bolotin A, Quinquis B, Sorokin A, Ehrlich SD. Clustered regularly interspaced short palindrome repeats (CRISPRs) have spacers of extrachromosomal origin. Microbiology 2005; 151: 2551-61.

6 Makarova KS, Aravind L, Grishin NV, Rogozin IB, Koonin EV. A DNA repair system specific for thermophilic Archaea and bacteria predicted by genomic context analysis. Nucleic Acids Res 2002; 15: 482-96.

7 Makarova KS, Grishin NV, Shabalina SA, Wolf YI, Koonin EV. A putative RNA-interference-based immune system in prokaryotes: computational analysis of the predicted enzymatic machinery, functional analogies with eukaryotic RNAi, and hypothetical mechanisms of action. Biol Direct 2006; 16: 1-7.

8 Barrangou R, Fremaux C, Deveau H, Richards M, Boyaval P, Moineau S, Romero DA, Horvath P. CRISPR provides acquired resistance against viruses in prokaryotes. Science 2007; 23: 1709-12.

9 Brouns SJ, Jore MM, Lundgren M, Westra ER, Slijkhuis RJ, Snijders AP, Dickman MJ, Makarova KS, Koonin EV, van der Oost J. Small CRISPR RNAs guide antiviral defense in prokaryotes. Science 2008; 15: 960-4

10 Deltcheva E, Chylinski K, Sharma CM, Gonzales K, Chao Y, Pirzada ZA, Eckert MR, Vogel J, Charpentier E. CRISPR RNA maturation by trans-encoded small RNA and host factor RNase III Nature 2011; 31: 602-7.

11 Jinek M, Chylinski K, Fonfara I, Hauer M, Doudna JA, Charpentier E. A programmable dual-RNA-guided DNA endonuclease in adaptive bacterial immunity. Science 2012; 17:337: 816-21.

12 Jiang F, Doudna JA. CRISPR-Cas9 Structures and Mechanisms. Annu Rev Biophys 2017; 46: 505-29.

13 Bakhrebah MA, Nassar MS, Alsuabeyl MS, Zaher WA, Meo SA. CRISPR technology: new paradigm to target the infectious disease pathogens. Eur Rev Med Pharmacol Sci 2018; 22: 3448-52.

14 Strich JR, Chertow DS. CRISPR-Cas Biology and Infectious Diseases Applications. J Clin Microbiol 2018; 1307-18 doi: 10.1128/ JCM.01307-18

15 Citorik RJ, Mimee M, Lu TK. Sequence-specific antimicrobials using efficiently delivered RNA-guided nucleases. Nat Biotechnol 2014; 32: 1141-5.

16 Yin C, Zhang T, Qu X, Zhang Y, Putatunda R, Xiao X, , et al. In Vivo Excision of HIV-1 Provirus by saCas9 and Multiplex Single-Guide RNAs in Animal Models. Mol Ther 2017; 25: 1168-86.

17 Bella R, Kaminski R, Mancuso P, Young WB, Chen C, Sariyer $\mathrm{R}$, , et al. Removal of HIV DNA by CRISPR from Patient Blood Engrafts in Humanized Mice. Mol Ther Nucleic Acids 2018; 7: 275-282.

18 van Diemen FR, Kruse EM, Hooykaas MJ, Bruggeling CE, Schürch AC, van Ham PM,Imhof SM, Nijhuis M, Wiertz EJ, Lebbink RJ. CRISPR/Cas9-Mediated Genome Editing of Herpesviruses Limits Productive and Latent Infections. PLoS Pathog 2016; 30:e1005701.

19 Bloom K, Maepa MB, Ely A, Arbuthnot P. Gene Therapy for Chronic HBV-Can We Eliminate cccDNA? Genes (Basel) 2018; 12:pii: E207. doi: 10.3390/genes9040207.

20 Zheng Q, Bai L, Zheng S, Liu M, Zhang J, Wang T, Xu Z, Chen Y, Li J, Duan Z. Efficient inhibition of duck hepatitis B virus DNA by the CRISPR/Cas9 system. Mol Med Rep 2017; 16: 7199-204.

21 Chiu C. Cutting-Edge Infectious Disease Diagnostics with CRISPR. Cell Host Microbe 2018; 13: 702-4.

22 Gootenberg JS, Abudayyeh OO, Lee JW, Essletzbichler P, Dy AJ, Joung J, et al. Nucleic acid detection with CRISPR-Cas13a/ C2c2. Science 2017; 28: 438-42.

23 Zuo X, Fan C, Chen HY. Biosensing: CRISPR-powered diagnostics. Nat. Biomed. Eng 2017; 1:1-2

24 Chen JS, Ma E, Harrington LB, Da Costa M, Tian X, Palefsky JM, et al. CRISPR-Cas12a target binding unleashes indiscriminate single-stranded DNase activity. Science 2018; 27: 436-9. 